\title{
ON THE FORMATION AND PROPAGATION OF INTERSTELLAR JETS
}

\author{
Guillermo Tenorio-Tagle \\ Max-Planck-Institut für Physik und Astrophysik \\ D-8046 Garching bei München, F. R. G.
}

Summary. The flow that results from stationary supersonically converging conical streams is studied by means of two dimensional numerical calculations. Convergency leads to a "colimator" conical shock and this to the formation of an underexpanded hydrodynamical jet. The properties of the conical shock and of the collimated stream are summarized for a variety of initial conditions. The resultant jets are followed to large distances $\left(\geq 7.5 \times 10^{17}\right.$ $\mathrm{cm}$ ) away from the injection point, and are shown to develop a complicated structure that eventually approaches a stationary state. Stationary features such as crossing shocks are shown to evolve from working surface shocks, left behind the head of the jet to ensure self-collimation of the beam matter.

Transient, as opposed to stationary, supersonically converging conical streams are also shown to generate collimator shocks, and under special conditions, long-lasting hydrodynamical jets and bipolar flows. Such flows are shown to emanate from compact disc funnels, if they are suddenly inmersed into a thermal bath. These calculations suggest that in many cases, the outflow phenomena (e.g. HH objects, jets and bipolar molecular flows) may simply be signaling the disruption of the compact discs, out of which exciting stars form.

I. Hydrodynamical jets powered by stellar winds. The solution to stationary supersonically converging conical flows has recently been worked out both analytically (Cantó et al. 1988) and numerically (Tenorio-Tagle et al. 1988). The converging streams have been assumed to result from an originally isotropic stellar wind redirected at an oblique "inner" shock which, in steady state and in the presence of a density gradient (see Cantó 1980 and Cantó and Rodriguez 1980), acquires an ovoidal "bishops hat" configuration. The wind gas upon crossing the oblique inner shock, acquires a pressure $P_{0}$, the original pressure of the ambient gas (as required by the steady state solution), and is redirected to move towards the axis of symmetry, generating in this way a stationary supersonically converging conical flow. The boundary and initial conditions for the numerical calculations have assumed a constant velocity $\left(U_{0}\right)$, uniform density $\left(n_{0}\right)$ stream, in pressure equilibrium with the surrounding medium and supersonically converging towards the symmetry axis with an incidence angle $\Theta$ (see figures 1 and 2). Once the density of the incoming flow ( $\left.n_{0}\right)$ is defined, the width of the stream can be derived from the assumption that all ejected wind matter $\left(\dot{M}_{w n d}\right)$, is recollected by the inner shock. The stream has been continuously replenished at the bottom of the computational grid to model the stationary converging flow.

The stationary supersonic flow inmediately develops a steady density enhancement caused by convergency alone, and a sudden further compression is provided by a conical shock that forms near the symmetry axis. Both features can be identified in figure 1 by noticing first the well spaced isodensity contour lines, drawn across the incoming stream, and 
Table 1

$\begin{array}{cccc}\Theta & \beta & \begin{array}{c}\mathrm{U}_{j e t} \\ \mathrm{~km} \mathrm{~s}^{-1}\end{array} & \mathrm{~A} \\ & & & \\ 15 & 3 & 98.4 & 1.6 \\ 30 & 1.8 & \mathbf{8 4 . 9} & \mathbf{2 . 7} \\ \mathbf{4 5} & 1.5 & 68.3 & 3.3 \\ 60 & 1.8 & 49.3 & 3.5 \\ \mathbf{7 5} & 4.0 & \mathbf{2 5 . 0} & 3.6\end{array}$

Fig.2.

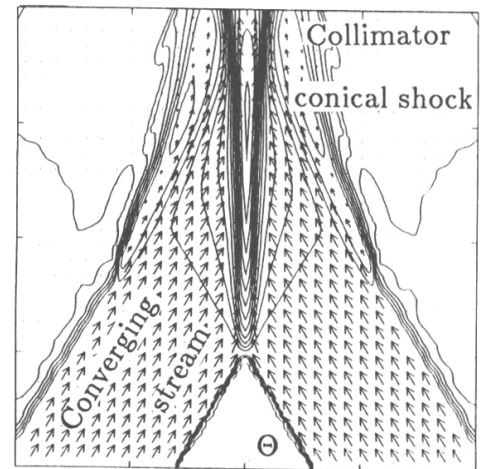

the surface (the conical shock) across which the density contours crowd and the velocity field is sharply redirected. The two features become almost inmediately stationary, regardless of the assumed incidence angle $\Theta$ (see Tenorio-Tagle et al. 1988). Note that the conical shock is also an oblique structure to the incoming stream and thus across it, the perpendicular component of the incoming velocity becomes thermalized while the parallel component is transmitted, bending the stream towards the shock, and defining the velocity of injection $\left(\mathrm{U}_{\text {jet }}\right)$ of the resultant flow. Strong radiative cooling (an isobaric process) leads also to a large compression behind the conical shock and in all calculated cases to a temperature of about $10^{4} \mathrm{~K}$. Such a temperature sets the value of the adiabatic speed of sound $\left(c_{j e t}\right)$ of the compressed matter before it is injected into the surrounding gas, and thus defines the Mach number $\mathrm{M}_{j e t}\left(=\mathrm{U}_{\text {jet }} / \mathrm{c}_{\text {jet }}\right)$ at the injection point. It is in fact $\mathrm{M}_{j e t}$, the one that accounts for the morphological sequence as a function of angle $\Theta$ (see figure 1), i.e. whether a thin elongated skinny fish or a fat french frog will result depends only on $\mathbf{M}_{j e t}$, as when the compressed gas exits the conical shock it inmediately expands into the surrounding gas. Its velocity of expansion is in all cases $c_{j e t}$ (about $10 \mathrm{~km} \mathrm{~s}^{-1}$ ), and thus the larger the $M_{j e t}$ the more elongated structures that will result.

The analytic and numerical results have shown that: i) the solution to a supersonically converging stationary conical flow is the formation of a stationary "collimator" conical shock, with its apex placed at the point of first contact. ii) In all cases where strong radiative cooling takes place (those with an incoming velocity $\mathrm{U}_{0} \cos \Theta<200 \mathrm{~km} \mathrm{~s}^{-1}$, which lead to large values of the interstellar cooling rate), the collimator conical shock presents a small aperture angle $\beta\left(<5^{\circ}\right.$, see figure 2 and table 1$)$. The solution to quasi-adiabatic cases can also be found in the paper that describes the numerical work (Tenorio-Tagle et al. 1988). iii) The velocity of the shocked gas $U_{j e t}$ is directed along the symmetry axis and thus given the small values of the aperture angle $\beta$, the relation $U_{j e t}=U_{0} \cos \Theta$, is a good approximation in all cases. iv) The incoming stream is compressed by a large factor $A\left(=\log \left(n_{j e t} / n_{0}\right)\right)$ which in all radiative cases amounts to more than two or three orders of magnitude (see Table 1). v) Radiative cooling behind the shock leads, in all cases, to a temperature $\mathrm{T}_{j e t}$ $\sim 10^{4} \mathrm{~K}$. This, together with the large compression factor results into a pressure $\mathrm{P}_{j e t} \gg$ $P_{0}$, and thus given (iii) the well directed velocity, it implies that the stationary collimator conical shock leads to the formation of an underexpanded $\left(\mathrm{P}_{j e t} \gg \mathrm{P}_{0}\right)$ hydrodynamical jet. Finally, vi) the radiative flow behind the collimator shock may be identified with the so called "optical jet structures", seen in the vicinity of some young stellar objects. 

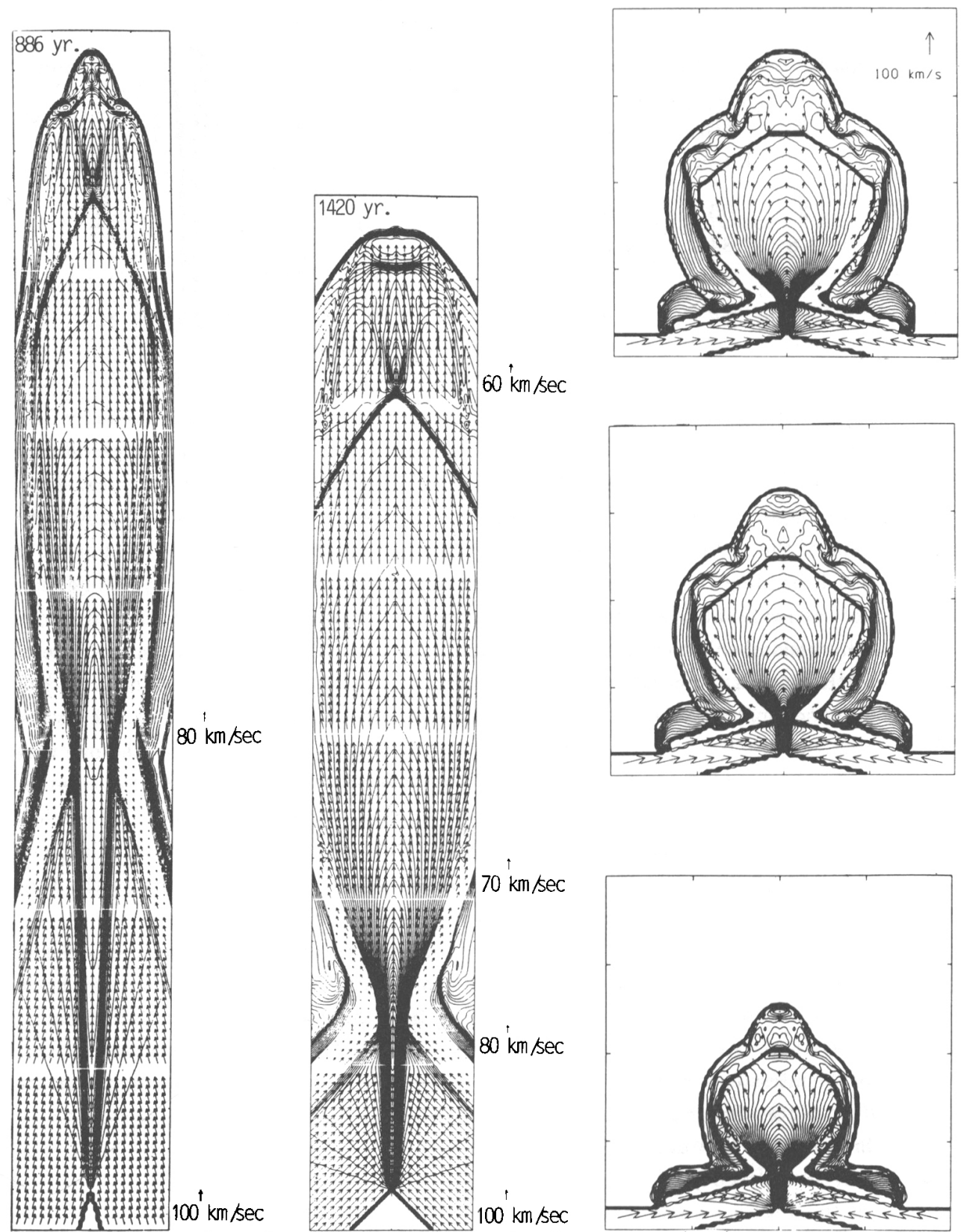

Fig. 1. Jet formation. Contours of equal mass density $\rho$ in the $(R, Z)$ plane are given for cases with $\mathrm{n}_{0}=100 \mathrm{~cm}^{-3}, \mathrm{U}_{0}=100 \mathrm{~km} \mathrm{~s}^{-1}$, and an incidence angle $\Theta=15^{\circ}, 45^{\circ}$ and $75^{\circ}$, respectively. The contour interval $\Delta \log \rho=0.2$ in the first two frames and 0.1 in the last case. The evolutionary times in $\mathrm{yr}$ are 886, 1420, and 1360, 2050, and 2320 for the $\Theta=75^{\circ}$ sequence. Superimposed is the velocity field to be compared with the standard velocity vector in $\mathrm{km} \mathrm{s}^{-1}$ given at the right hand side of the plots. Changes in the absolute magnitude of this vector are also indicated. The distance between consecutive tick marks is $10^{16} \mathrm{~cm}$ in the first two frames and $4 \times 10^{16} \mathrm{~cm}$ in the $\Theta=75^{\circ}$ panels. 
II. The propagation and structure of interstellar jets.

Figure 3 displays a fully developed hydrodynamical jet, produced by the mechanism described in section $I\left(n_{0}=100 \mathrm{~cm}^{-3}, U_{0}=100\right.$ $\mathrm{km} \mathrm{s}^{-1}, \Theta=30^{\circ}$, initially in pressure equilibrium with an outside medium where $\mathrm{n}=10^{3} \mathrm{~cm}^{-3}$ and $\mathrm{T}=10 \mathrm{~K}$ ), after some $7000 \mathrm{yr}$ of evolution. Perhaps, it is the time dependent jet with the largest length to width (at injection point) ratio, so far calculated in an astrophysical context. A full description of its density, temperature and pressure structure as it propagates through interstellar space is to appear in a forthcoming paper (Tenorio-Tagle and Różyczka 1989). Here only a brief summary of the results is given. Before that however, one should make the point that the structure left by the jet as it propagates away from the injection point is independent of the formation mechanism. i.e. once an overpressured, preferentially well directed supersonic beam forms, by whatever means, and it is injected into the surrounding gas, it will propagate while digging a channel and causing a structure similar to the one shown in figure 3 . How does such a structure develop? and/or how is collimation at all distances from the injection point achieved? are some of the main issues presently in this field.

As mentioned in section $I$, as soon as the collimated matter is injected into the surrounding gas, it beyins to expand. Expansion is due to the overpressure $\left(\mathrm{P}_{j e t} \gg \mathrm{P}_{0}\right)$, which causes the divergency of the beam at a speed $c_{j e t}\left(\sim 10 \mathrm{~km} \mathrm{~s}^{-1}\right)$. As matter moves away from the injection point its density drops as $1 / R^{2}$, and it cools down both by radiation and by its own expansion. Thus, the diverging beam eventually reaches pressure values which are even lower than $P_{0}$. However, its supersonic motion leads to the establishment of another oblique shock (an "incident" shock) across which the beam pressure is restored. Across any oblique shock, only the component of the gas velocity perpendicular to the shock becomes thermalized and enhances the pressure. The other component of the velocity (the one parallel to the shock), is almost fully transmitted and thus the gas is effectively deflected towards the shock. The first of these properties defines the shape of the incident shock, which extends across to overtake the whole expanded beam, while giving it the same pressure $\left(\mathrm{P}_{\text {shock }}\right)$. Clearly the expanded beam matter closer to the injection point retains a larger value of the original pressure and would meet a section of the incident shock which is almost parallel to its own motion, and thus hardly modifies its pressure. However, rarer and

Fig. 3. The structure of interstellar jets. The same as figure 1 for a stream converging with an incidence angle $\Theta=30$, after 7700 $\mathrm{yr}$ of evolution. The distance between consecutive tick marks is $10^{16}$ $\mathrm{cm}$. Some of the contours are labelled with the value $\log \rho\left(\mathrm{g} \mathrm{cm}^{-3}\right)$.

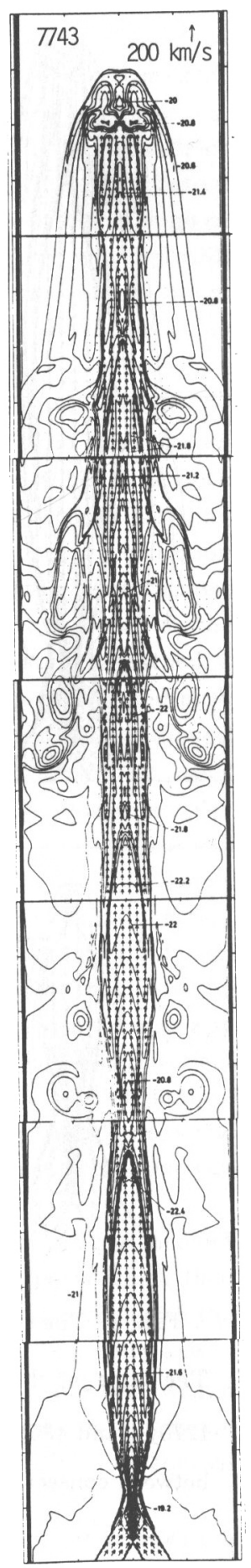




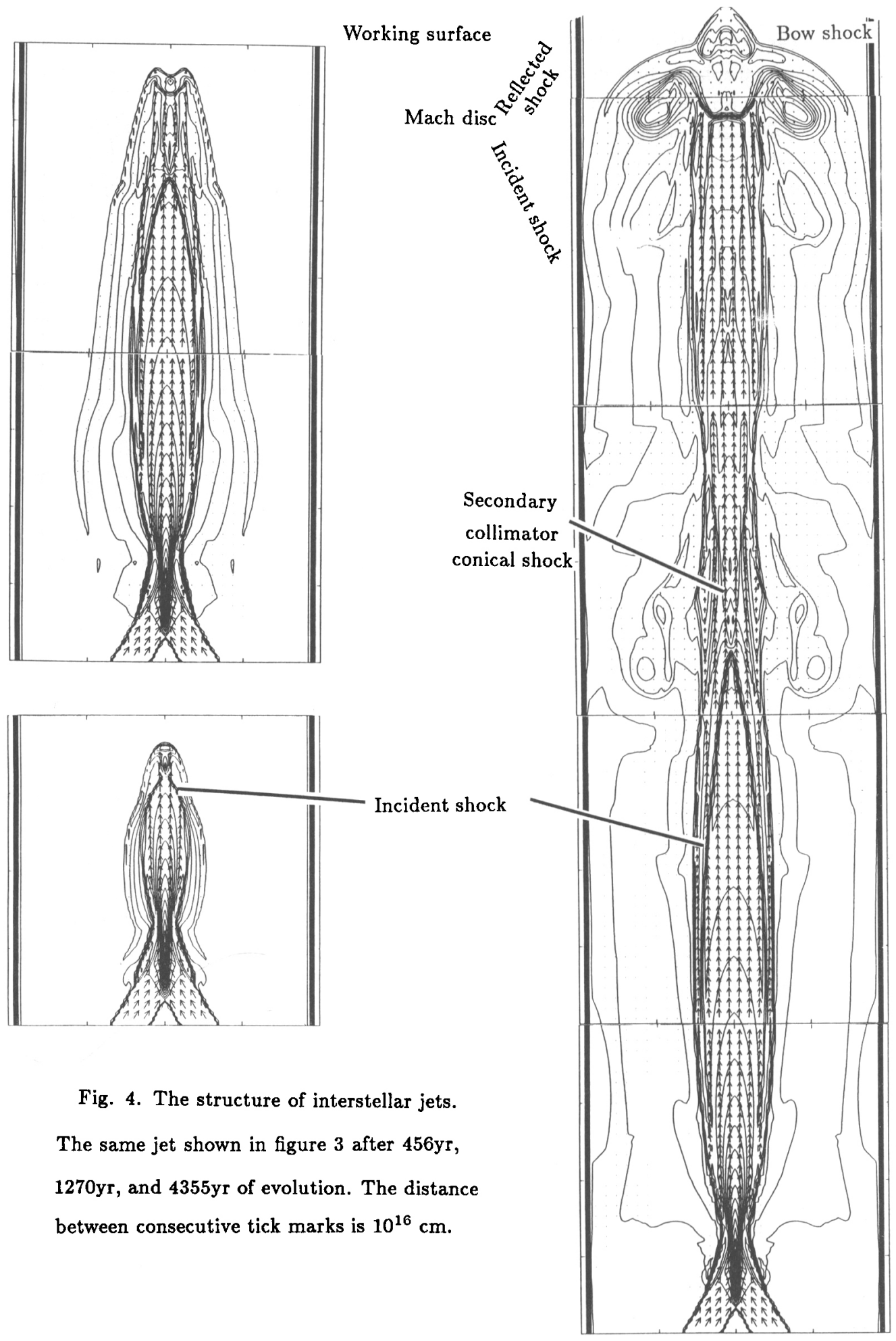


further away beam matter, will cross a more frontal section of the incident shock in order to acquire the same pressure value $\left(P_{\text {shock }}\right)$. Meanwhile, the shocked bearn is redirected to slide behind the incident shock. As long as $P_{\text {shock }}$ is larger than $P_{0}$, the incident shock will continuously adjust, mainly by moving further away from the injection point, until the steady state condition, $\mathrm{P}_{\text {shock }}=\mathrm{P}_{0}$, is achieved. At that moment, the incident shock becomes a standing feature in the flow, and the shocked matter behind it evolves into a stationary supersonically converging conical stream. The solution to such a flow is given in section I. Clearly, a secondary collimator conical shock will form. This will replenish the beam and will re-inject it into the surrounding gas, mimicking our initial condition. Therefore, the flow pattern is to repeat itself, many times, as the head of the jet continues to penetrate the interstellar gas.

Figure 4 shows the same jet shown in figure 3, at earlier times. The two features the incident shock and secondary collimator conical shock (referred to usually as the reflected shock) are clearly indicatcd. Notice however that at such former evolutionary times, both structures continuously move with the flow. Furthermore, both features are originally sitting at the head of the jet, and in fact form part of the so called working surface. i.e. a collection of incident, reflected and Mach disk shocks, which while decelerating the beam matter, provide it with the large overpressure that drives a leading bow shock. $\Lambda \mathrm{s}$ soon as the reflected shock begins to replenish the beam, even before its stationary state is reached, a new set of working surface shocks appears to lift the pressure of the beam matter and push further the leading bow shock. Such an evolution therefore also causes drastic temporal changes at the head of the jet which in fact become periodic as the jet grows. i.e. they repeat themselves every time the head of the jet crosses a location to be eventually occupied by a stationary set of incident and reflected shocks. Figure 4c displays for example a well developed set of working surface shocks with a wide Mach disk joining at triple points the new set of incident and reflected shocks. The Mach disk presents its maximum extent when the working surface lies half-way between the last collimation point and the future stationary location of the incident shock. It then decreases in size, to become unresolved by the numerics, as the incident shock approaches its new stationary state. Such changes also affect the shape, and thus the strength, of the leading bow shock. Therefore, the large variety of strengths and possible shapes acquired by the shocks at the head of a hydrodynamical jet could certainly explain the variety of degrees of excitation observed in IIII objects, while accounting for their observed proper motions. From the study of the jet structure one can conclude that:

i) In an underexpanded jet, self-collimation is achieved by means of oblique incident shocks which drive the beam matter to compose converging conical streams which then lead to the appearance of reflected (secondary collimator conical) shocks that replenish the beam, provide it with an overpressure, and re-direct it to move along the symmetry axis.

ii) Every pair of incident and reflected shocks form a so called "crossing" shock within the jet structure. The time dependent solution has shown us that crossing shocks are evolved working surface shocks that approached a stationary state to provide the required self collimation.

iii) The separation between stationary crossing shocks depends only on the value of the initial ambient pressure $P_{0}$. When the pressure behind an incident shock $P_{\text {shock }}=P_{0}$, the 
crossing shock becomes stationary and thus, the larger the initial pressure $\mathrm{P}_{0}$ the smaller the separation.

iv) Several crossing shocks may be found along a jet structure. However, from these only the most outer ones would be moving, adjusting the flow to become stationary. Thus, their relation to the emitting knots seen in optical jet structures near young stellar objects may be an over-interpretation of the theory. Note for example that the measured velocity of observed neighbouring emitting knots is vastly different, and both accelerating as well as decelerating velocity patterns have been detected (Reipurth, 1989).

\section{Jets and outflows driven by a thermal bath.}

No doubt nature provides several other ways of generating supersonically converging conical streams, and thus a variety of events capable of producing hydrodynamical jets. This section of the paper deals with a mechanism, and the range of parameters required, to cause a jet from the conical supersonic convergence of matter originally belonging to a compact disc (CD). The project, presently being carried out in collaboration with Drs. J. Franco and $\mathrm{P}$. Bodenheimer, assumes a radiative flux produced by a recently formed star, or at its accretion shock, that strongly modifies the temperature structure of the $\mathrm{CD}$. Upon perturbation of the disc, large pressure gradients result and these while overwhelming the forces that originally keep the disc together, lead to a strong acceleration of the irradiated matter. For the case of massive stars, Bally and Scoville (1982) and Pudritz (1985) have suggested that supersonic outflows should result upon photo-ionization of the disc matter out of which the stars formed. Photo-ionization leads to a temperature $\left(\mathrm{T}_{\text {gas }}\right)$ of about $10^{4} \mathrm{~K}$, and this to strong pressure gradients, both at the border of the HII region (at the ionization front) and within the ionized volume, between all neighbouring parcels of gas presenting strong density gradients The pressure gradients lead then to expansion, both into the remaining neutral section of the disc, causing a strong compression of the neutral matter, and into the lower density sections of the HII region volume. The latter (as shown by Tenorio-Tagle, 1979) leads to supersonic motions directed along the initial density gradients, and cause the rapid disruption of the $C D$ (see also the contribution of Dr. Franco). Thus, a crucial issue in such a model is the true density stratification of a CD. A self consistent approach should account for rotation, the central gravitational force, the presence of a $B$ field and for the thermal pressure of the CD. Such a detailed structure is beyond the present initial approach to the problem. Instead, adhoc density distributions, similar to those shown in figure 5 have been assumed. Such density stratifications present two regimes: sections of the disc, far from the central source, have a plane stratification with a constant scale height $(\mathrm{H})$. Closer to the center, the distribution may still be described with a plane stratification but with a decreasingly smaller scale height, causing a well defined funnel with a decreasingly smaller aperture angle $\alpha$, as one approaches the axis of symmetry.

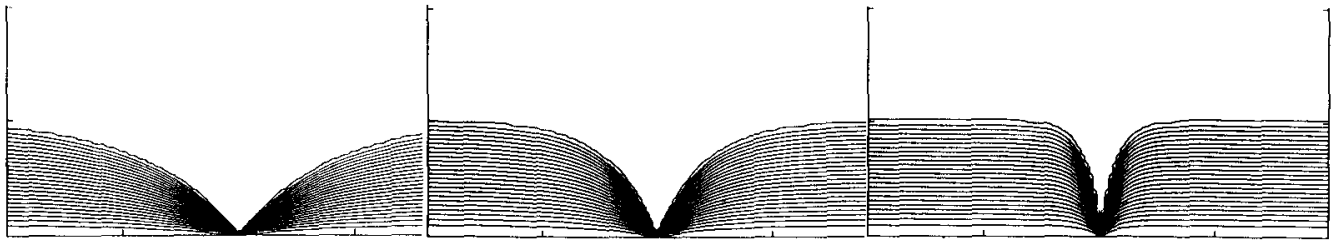

Fig. 5. Adhoc CD density distributions 
Note that such an stratification may not be identical to the actual true density distribution in $\mathrm{CDs}$, however our approximations are likely to cover the range of parameters representing the true aspect of CDs. Given such a density distribution, upon photo-ionization the pressure gradients within the ionized volume would lead to two well directed outflows: one of them, perpendicular to the disc plane, would emanate from the ionized section of the disc with an initial constant H. A second faster flow, would develop from the disc funnel where the density gradient is even more abrupt. This latter flow will clearly be a supersonic conical stream, converging towards the symmetry axis with an incidence angle $\Theta$, pre-set by the local aperture of the $\operatorname{CD}(\Theta=90-\alpha)$. Figure 6 shows two dimensional calculations of these flows. In both cases the original density at the disc plane is $10^{6} \mathrm{~cm}^{-3}$ while outside the interstellar matter density is $1 \mathrm{~cm}^{-3}$. The ionizing flux $\left(F_{\star}\right)=10^{49}$ photons $s^{-1}$, and the ionized section of the discs is indicated in the figures by a dashed line. Expansion leads to large velocities and thus within a few hundred years the irradiated disc is disrupted. The largest velocities occur at the skin of the disc, where the density gradient is largest however, the whole outward flow becomes supersonic $\left(u \gg 10 \mathrm{~km} \mathrm{~s}^{-1}\right)$ and soon it reaches large dimensions (see figure 6).
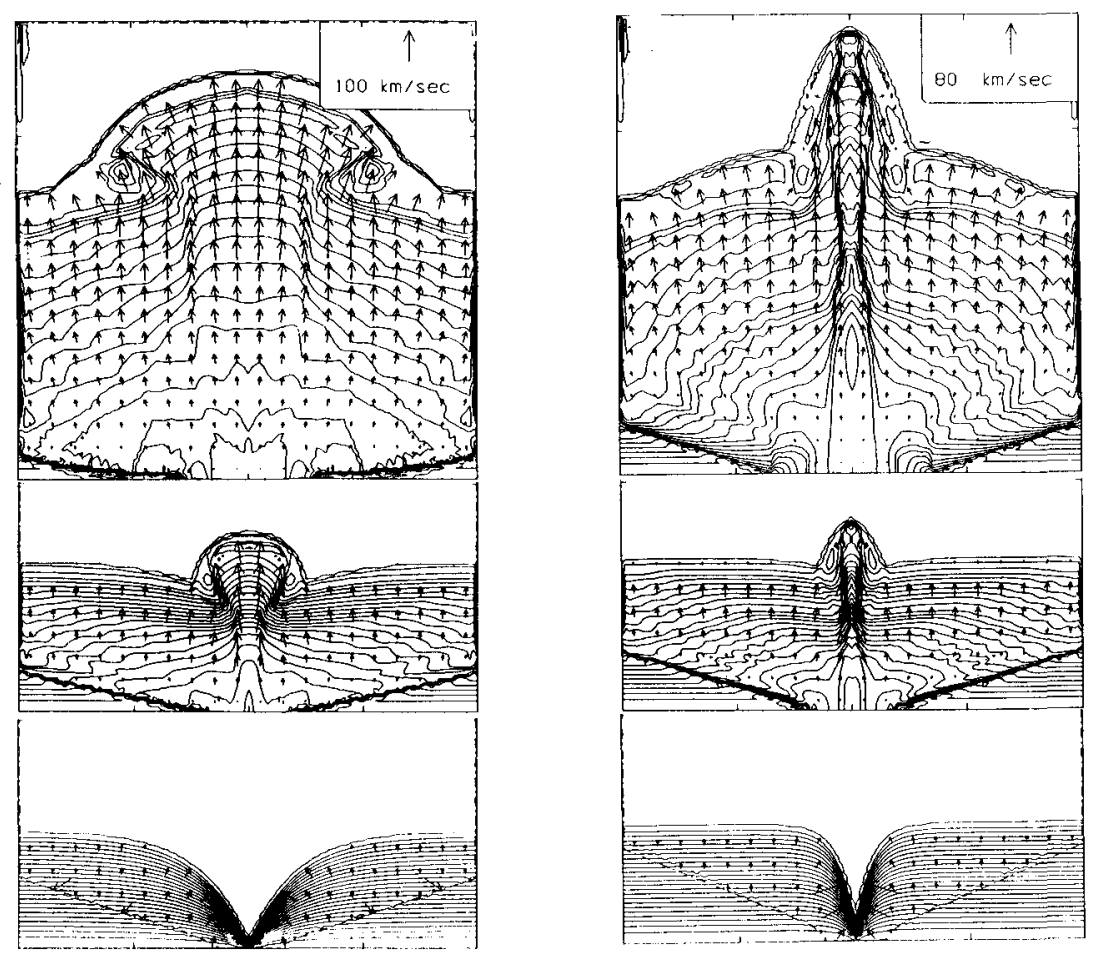

Fig. 6. CD disruption. Similar to figure 1, The original CD density stratification and evolution of two cases are shown at times $147 \mathrm{yr}, 522 \mathrm{yr}, 1089 \mathrm{yr}$, and $150 \mathrm{yr}, 814 \mathrm{yr}$, and $1453 y r$, respectively. Distance between consecutive tick marks is $7.5 \times 10^{16} \mathrm{~cm}$. 
All models for the formation of either jets, $\mathrm{HH}$ objects and/or molecular outflows when confronted with the observations present energy problems. This is also the case when applying the above results to the outflows seen in the vicinity of low mass stellar objects. In such a case, surely both the IAU and some of the respectable members of the establishment, will not allow the usage of the expensive ionizing radiation $\left(F_{*}\right)$ assumed in the above calculations, and therefore several steps have been taken to alleviate the energy problem. First of all, the photon flux has been reduced by six orders of magnitude. Note that an $\mathrm{O} 6$ star produces $10^{49}$ photons $s^{-1}$, while a B0.5 has an output of $10^{46}$ ionizing photons $s^{-1}$ (see Osterbrock 1989). In the following calculations $F_{\star}$ has been set equal to $10^{43}$ photons $s^{-1}$.
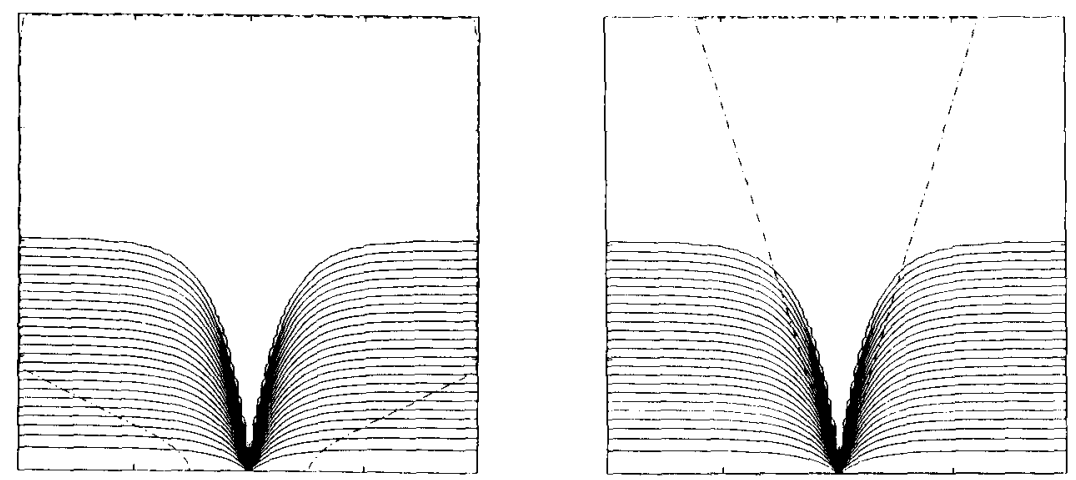

Fig. 7. The irradiated volume. The initial HII region volume caused by an $F_{\star}=10^{49}$ and $10^{43}$ photons $s^{-1}$ are shown. Distance between consecutive tick marks is $3.75 \times 10^{16}$ cm.

Such a flux causes a much smaller ionized initial volume as indicated in figure 7. Secondly, the temperature caused by the photon flux, has been set to $\mathrm{T}_{g a s}=2000 \mathrm{~K}$, instead of the $10^{4} \mathrm{~K}$ resultant from photo-ionization. This assumes that the average energy per photon is smaller than the $13.6 \mathrm{eV}$ required to ionize $\mathrm{H}$, and may lead to photo-dissociation only. How many of these photons are produced at the surface of a young stellar object or at its accreation shock, say when it undergoes a $\Theta$ Orionis phenomena, is not known and thus it has been set equal to $10^{43}$ photons $s^{-1}$. A further step in this direction has been to simulate a short flash of radiation, rather than the constant photon flux condition used in the case of massive stars. This has been achieved by means of a boundary condition which sharply cuts the photon flux after a pre-set critical time $\left(t_{c r i t}\right)$. e.g. $F_{\star}=F_{\star}$ (time $/ t_{c r i t}$ )$^{-5}$. Values of $\mathrm{t}_{\text {crit }}$ equal to $100 \mathrm{yr}, 20 \mathrm{yr}$, and $5 \mathrm{yr}$ have been used without leading to noticible changes in the resultant flows. The calculation shown in figure 8 resulted from an $F_{\star}=10^{43}$ photons $s^{-1}$, which provided the irradiated matter with a temperature $\mathrm{T}_{g a s}=2000 \mathrm{~K}$, and was sharply reduced after a $t_{c r i t}=5 y r$. The irradiation of the disc leads to similar flows to those obtained for the case of massive stars (compare figures 8 and 6). The irradiated funnel responds inmediately to the pressure gradient and collapses supersonically towards the symmetry axis. The self-collision starts at the "surface" of the star causing the formation of a collimator shock. This however, given the finite amount of converging matter, cannot be supported at such location and thus it effectively moves upwards along the symmetry 
axis, with a phase velocity $U=U_{0} / \cos \theta$, as matter originally further away approaches it with a velocity $U_{0}$. The collimator shock presents under these circunstances a narrow cylindrical shape the length of which is determined by the local duration of the transient converging flow, and the phase velocity $U$. The cylindrical shock is also an oblique shock to the incoming funnel matter. Note that given the continuous change in the local value of $\alpha$ the shocked matter would retain larger values of the incoming velocity, the further away from the stellar surface that it encounters the cylindrical shock. Concecuently, the velocity of the collapsing material originally deep inside the funnel, where the aperture angle $\alpha(=\alpha$ 0 ) is small, will be almost fully termalized leading, upon radiative cooling, to a dense rod of shocked matter slowly moving along the symmetry axis. It is in fact not until matter with an original angle $\alpha \geq 45^{\circ}$ reaches the cylindrical shock that a fast well directed stream ( $U_{j e t}$ $=\mathrm{U}_{0} \cos \Theta$ ) begins to be injected into the undisturbed surrounding gas, pouring out of the tip of the cylindrical collimator shock. The shock is still moving upwards with its phase velocity $U$. However by then, $U$ has become smaller than the transmitted velocity $U_{j e t}$, allowing for the injection of the well directed supersonic beam and thus for the formation of a hydrodynamical jet (see figure 8). Not all density distributions lead to such feature (see figure 6). If the original aperture angle $\alpha_{0}$, at the deepest part of the funnel is large (say $\geq 45^{\circ}$ ), then the converging flow has time to fan away even before it reaches the cylindrical shock, and thus inhibiting the formation af a jet. Cases with a small $\alpha_{0}$ (see figures 6 and 8) lead to a better collimation.

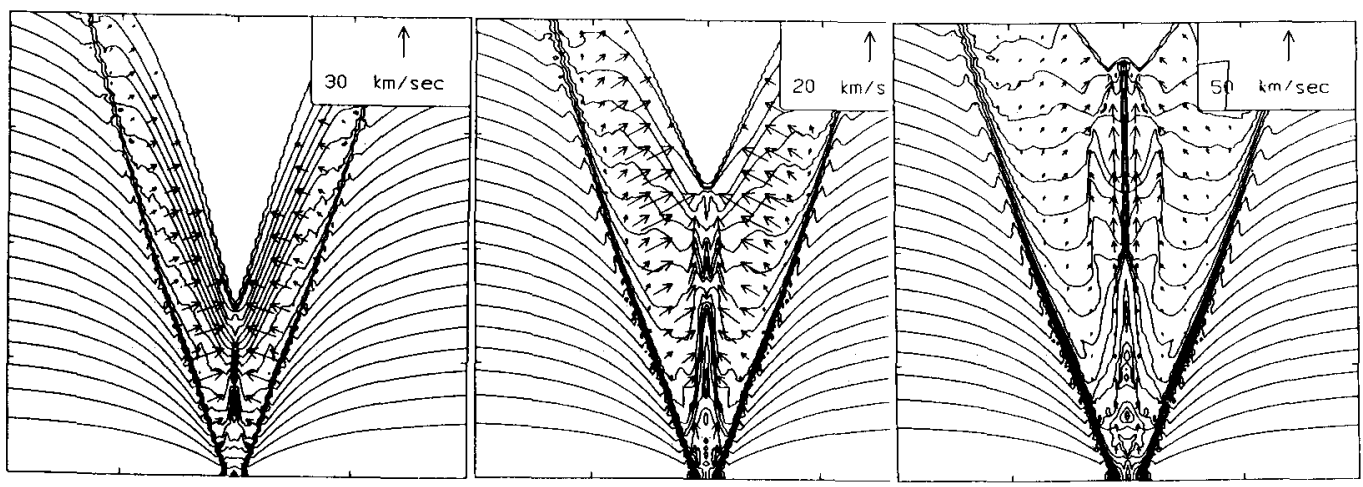

Fig. 8. The supersonic collapse of the CD funnel. A time secuence obtained at $t=$ $94 \mathrm{yr}, 140 \mathrm{yr}$, and $200 \mathrm{yr}$, showing the rapid development and upward motion of the cylindrical collimator shock. The last frame also shows the collimated flow begining to move ahead of the converging conical stream. Distance between consecutive tick marks is $1.5 \times 10^{16} \mathrm{~cm}$.

The fact that all calculated flows look alike under all circunstances considered i.e. under the assumption of photo-ionization or photo-dissociation, and even in the cases where a short flash of radiation was assumed, result from the lengthly recombination and cooling times both of which are much larger than the dynamical time promoted by the pressure gradients. Thus, even if the densest parts of the irradiated disc cool fairly quickly, by then the flow pattern would be fully developed. i.e. matter may cool and/or recombine however, as the momentum gained is not radiated away, the cooling stream will continue to 
move supersonically along the track defined by the pressure gradients. Therefore, from the explored parameter space which include the CD density distribution, the value of $F_{*}$, its duration, and the temperature acquired by the irradiated gas $\left(\mathrm{T}_{\text {gas }}\right)$, it seems likely that bipolar flows, jets and their resultant $\mathrm{HH}$ objects could in many cases be simply signaling the disruption of the $C D$, remnant of the stellar formation process. The disruptive radiation may arise from either the star or from its accretion shock, but all irradiated sections of the CD would be disrupted leading to well directed outflows. The event could in principle be repeated many times in the same source, if the remains of the disc have time to readjusts before another massive accretion event takes place.

The model for jet formation here described differs from all other models that suggest the funnel matter to be mechanically pushed aside until a nozzle forms and a rarer interior gas streams through. Instead, these calculations have shown the unavoidable supersonic collapse of the disc funnel, which in some favorable cases leads to the formation of a hydrodynamical jet, upon the sudden inmersion of the disc matter into a thermal bath.

Acknowledgments.

I am gratefull to Drs. M. Różyczka, J. Franco and P. Bodenheimer for allowing me to show some of our results prior to publication.

\section{References}

Bally, J. , Scoville, N. Z. 1982.: Astrophys. J.255,497.

Cantó, J.: 1980, Astron. Astrophys. 86, 327

Cantó, J., Rodriguez, L.F.: 1980, Astrophys. J 239, 982.

Cantó, J., Tenorio-Tagle, G., Różyczka, M.: 1988, Astron. Astrophys. 192, 287

Osterbrock, D. E. 1989. Astrophysics of Gaseous Nebulae and Active Galactic Nuclei.

University Science Books. Mill Valley, California. p 22.

Pudritz, R. E. 1985.: Astrophys. J. 293,216.

Reipurth, B.: 1989, Astron. Astrophys. (in press)

Tenorio-Tagle, G. 1979, Astron. Astrophys. 71, 59.

Tenorio-Tagle, G., Cantó, J., Róźyczka, M.: 1988, Astron. Astrophys. 202, 256

Tenorio-Tagle, G., Różyczka, M.: 1989, Astron. Astrophys. (in press) 


\section{Discussion:}

ELSÄSSER. What one observes in several cases, for instance in L1551, is a rather broad flow pattern besides a strongly collimated jet within this. Can this phenomenon be understood within the frame of your model?

TENORIO-TAGLE. First of all allow me to say that in observational astronomy everything that appears luminous and straight is called a jet. A true hydrodynamical jet must present the structure discussed in section II, otherwise it is not a jet. I do see great resemblance between the flows detected in several sources and the computed models shown in section III (see figures 6-8). A well developed flow emanating from the disrupted CD contains an elongated, straight and denser rod of matter, formed during the collapse of the deepest part of the CD funnel. This, given its larger density cools faster and thus it should appear more luminous than the overall flow, in agreement with your observations.

DYSON. What happens if you include stellar winds?

TENORIO-TAGLE. As shown in figures 6-8, the thermal bath leads to a supersonic motion and thus within a few hundred years (see figure 8) the flow or thermal wind, which in some cases may be interpreted as a stellar wind!, is well established. Therefore I will expect that by the time your winds become important, if at all, the whole density distribution will already be largely modified by the thermal disruption of the $C D$. This is particularly true for all models based on the steady state wind configuration which requires a much longer time to be reached.

PECKER. You obtain splendid collimation! But you start from a strictly symmetrical cylindrical geometry. Would the actual initial condition be less strictly regular, let us say with fluctuations in density distribution, would you keep the collimating effect, or on the contrary, would this collimating effect be entirelly destroyed?

TENORIO-TAGLE. All models here described for the formation of hydro jets do required a supersonic conical stream converging towards the symmetry axis. I suppose, slight deviations in the symmetry of this flow could still yield a jet.

MELNICK. When you look at detailed narrow band optical pictures of jets you can get the impresion that they have helical structures that, at least to me, suggest the presence of magnetic fields. Can you comment on how would magnetic fields modify your model?

TENORIO-TAGLE. Perhaps one should wait for a definite confirmation before jumping into $B$ field wild speculation. In the mean time I shall try to cure my present phobia agains magnetic fields. 